\title{
Determining the efficiency of photoelectrode Materials by Coupling Cavity-Microelectrode Tips and Scanning Electrochemical Microscopy
}

\author{
Alberto Visibile, ${ }^{a}$ Tomasz Baran, ${ }^{b}$ Sandra Rondinini, ${ }^{a, c}$ Alessandro Minguzzi, ${ }^{a, c *}$ Alberto Vertova, ${ }^{a, c}$ \\ [a] Dr. A. Visibile, Professor S. Rondinini, Professor A. Vertova, Professor A. Minguzzi \\ Laboratory of Applied Electrochemistry, Dipartimento di Chimica, \\ Università degli Studi di Milano \\ Via Golgi 19, 20133, Milan, Italy \\ alessandro.minguzzi@unimi.it \\ [b] Dr. Tomasz Baran \\ SajTom Light Future, \\ Wężerów 37, 32-090 Wężerów, Poland \\ [c] Professor S. Rondinini, Professor A. Vertova, Professor A. Minguzzi \\ Istituto Nazionale di Scienza e Tecnologia dei Materiali \\ Via Giusti 9, 50121, Florence, Italy
}

\begin{abstract}
:
Photoelectrochemical water splitting (PEC-WS) is a promising route to obtain hydrogen (and oxygen) from sunlight and water. However, too many semiconductors show poor stability, due to photodegradation phenomena in aqueous solutions, thus loosing efficiency under operative conditions.
\end{abstract}

Aim of this paper is to introduce a simple and fast method for screening different semiconductor materials and identify their efficiency in $\mathrm{H}_{2}$ (or $\mathrm{O}_{2}$ ) production with respect to photocorrosion. This method could be used with any finely dispersed semiconductor (powder) for a fast, preliminary evaluation of the material's behaviour without interferences from the supporting material (i.e FTO) or any binder.

The method is based on the combination of scanning electrochemical microscopy (SECM) in the tip generation /substrate collection (TG/SC) mode and of cavity microelectrodes as SECM tips. In this paper we show results obtained on three powder materials, namely coreshell $\mathrm{Cul} / \mathrm{CuO}, \mathrm{Cul}$ and $\mathrm{TiO}_{2}$.

\section{Introduction:}

The efficient exploitation of renewable energy source is one of the main limiting factors towards a sustainable development of human activities. In this context, the use of hydrogen as energy vector is one of the most promising routes. In photo-electrochemical water splitting, $\mathrm{H}_{2}$ can be directly obtained through water electrolysis assisted by solar energy. p-type and n-type semiconductors are used as photocathode and photoanode light capturing materials, respectively.

An ideal semiconductor for PEC-WS should possess the following characteristics: i) be abundant, low-cost, and nontoxic, ii) have a narrow band gap iii) have the correct bands position iv) be stable in aqueous media and under working conditions. Most frequently, the choice is based on a compromise between stability and activity, the latter depending on the range of frequencies that the semiconductor can absorb. For example, titanium dioxide has a sufficient stability but its wide band gap (around 3.2 eV) excludes the absorption of visible light ${ }^{[1-5]}$. On the other hand, materials like $\mathrm{WO}_{3}, \mathrm{Fe}_{2} \mathrm{O}_{3}, \mathrm{BiVO}_{4}$ or $\mathrm{Cu}(\mathrm{I})$ and $\mathrm{Cu}$ (II) oxides can absorb part of the visible spectrum but are still under characterization in terms of stability and performances. ${ }^{[6-13]}$.

Figure 1 summarizes the different processes occurring after the absorption of a photon in a semiconductor material immersed in an (aqueous) electrolyte. The desired charge transfer phenomena (reaction IV in Fig. 1) to produce hydrogen (or oxygen) are not the only possible pathways for photogenerated electrons and holes: once these are close to the corresponding energy band edges they can undergo non-radiative and radiative 
recombination (reaction II in Fig. 1) and reactive and nonreactive trapping ${ }^{[14]}$ (reaction I in Fig. 1) by surface sites. These undesired paths reduce the efficiency of sunlight to $\mathrm{H}_{2}\left(\right.$ and $\mathrm{O}_{2}$ ) conversion.

Moreover, the main cause of material degradation is the electron (or hole)-transfer reaction to the material itself with consequent loss of activity, due to photodegradation/photocorrosion (reaction III in Fig. 1). This process is different from the electron-hole recombination where the energy is usually lost as heat. Here, the electron (or hole), can lead to a reduction (or oxidation) of the semiconductor itself. Such process does not occur on any semiconductor, but low/intermediate band gap photocathodes (e.g. $\mathrm{ZnS}, \mathrm{CdS}, \mathrm{Cu}_{2} \mathrm{O}$ ) typically tend to be reduced to the corresponding metals. This photodegradation reaction is even stronger for very pure materials suggesting the importance of doping ${ }^{[15]}$ and occurs every times the redox potential of the material's redox reactions lie within the band gap. Many strategies can be applied to improve the stability of materials e.g. metal or non-metal doping, surface modifications by metallic co-catalyst or by interaction with plasmonic metals $^{[16]}$

Indeed, for a photocathode, self-reduction processes are very likely leading to a loss of photoactivity of the material due to the formation of a thin film of non-active metal. In the case of a well-known photocathodes like $\mathrm{Cu}_{2} \mathrm{O}$, this reaction leads to the formation of metallic $\mathrm{Cu}$. No evidence of $\mathrm{Cu}_{2} \mathrm{O}$ oxidation were achieved with the use of $\mathrm{XAS}^{[17]}$, meaning that there is no self-oxidation of the material induced by free holes generated in the material, but this process can still occur on other photoactive materials ${ }^{[18-23]}$.

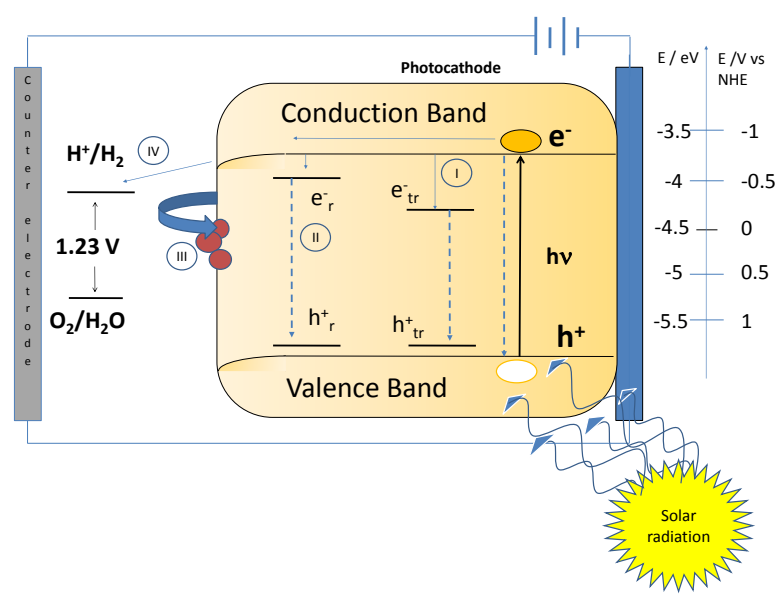

Figure 1. Schematic representation of the different processes occurring in a photocathode under solar radiation after the electro-hole couple generation.
I) trapping; II) electron-hole recombination; III) material photodegradation with formation of photocorroded material on the surface; IV) water reduction.

It is very hard to distinguish and quantify all undesired contributions from the photocurrent related to the desired process. Indeed, simply considering the photocurrent obtained with a given material under working condition is meaningless for the evaluation of the electrode performances because it is impossible to extract the portion relevant to water splitting. A high current may indeed be mostly related to material photodegradation rather than to a good performance in water reduction/ oxidation.

As a parallelism with the well-known faradaic efficiency, widely used in electrochemistry, we can define the photofaradaic efficiency as the ratio between the photocurrent related to hydrogen (or oxygen) production and the total photocurrent:

$\phi_{P E C}=\frac{I_{H_{2}}}{I_{H_{2}}+I_{\text {photodegrad }}}$

Where $I_{H_{2}}$ is the HER related photocurrent, and $I_{\text {photodegrad }}$ is the part of photocurrent connected to the undesired process of photodegradation.

Being able of quantifying the intensity of any undesired degradation processes would allow to correctly choose the best candidates for long-term and efficient PEC-WS system.

In the present work, we propose a novel method based on the combined use of the scanning electrochemical microscopy (SECM) in the tip generation/substrate collection mode (TG/SC) and cavity micro-electrodes $(\mathrm{C}-\mathrm{MEs})^{[24,25]}$ for the rapid screening of semiconductor photoactivity and for the determination of the relevant photocurrent efficiencies. The method here described can be applied easily to all the powder materials of interest thanks to the use of C-ME able to accommodate the material itself; it is an in-situ method allowing for real time characterization.

This was achieved by using a SECM with a C-ME (instead of conventional microdisk tips) filled with the desired $n$ - or p-type semiconductor powder, and approaching at selected distance a flat Pt substrate. In turn, the latter detects the $\mathrm{H}_{2}$ produced at the tip. The tip/substrate gap can be illuminated by means of a $3.5 \mathrm{~W}$ LED and the tip potential is controlled at will. A sufficiently large Pt substrate (about 
5 orders of magnitude higher than the tip/substrate gap) guarantees that all the $\mathrm{H}_{2}$ produced at the tip is oxidized at the substrate (unitary collection efficiency), as proposed for studying multireactional electrochemical interfaces ${ }^{[26]}$.

It follows that substrate and tip currents will be equal only if the sole product of the photoelectrochemical process at the tip is $\mathrm{H}_{2}$ evolution. Otherwise, the two currents will differ, most likely because photocorrosion phenomena, charge recombination or, in rarer cases, production of soluble products not detectable by the substrate.

The most adopted methods for evaluating $\phi_{P E C}$ include gas chromatography and volume liquid displacement ${ }^{[27-30]}$. These techniques have rather big disadvantages, i.e. the complexity of the experimental procedure (e.g. calibration) and its relatively low precision (about $13 \%$ error) ${ }^{[6]}$ for volume displacement. In addition, GC gives no evidence on the nature of the gas evolved ${ }^{[6]}$, unless standards are used. Mostly important, GC is not a time-resolved technique.

The proposed protocol, that combines SECM and C-ME tip, is far more rapid and offers the possibility to obtain information on the material's efficiency in a wide range of applied potentials (using a single electrode) in a few minutes. Moreover, being time resolved, the here introduced method is particularly interesting for the study of unstable materials that do not show a constant efficiency.

In addition to its fast screening features, the present method is not affected by any contribution from the supporting material (i.e. FTO or ITO) and of binders that might introduce artifacts: this is one of the most recognized features of C-MEs ${ }^{[31-36]}$.

Other advantages of C-ME, e.g. the precise control of the amount of powder loaded in the cavity, are well established $^{[24,25,31,36]}$, and the combined use with SECM was previously introduced as well in the case of high conductivity powders for the OER ${ }^{[37]}$. The use of Au CM-E's filled with the studied material is equivalent to preparing custom microdisk tips, avoiding time consuming techniques for the preparation of microdisks of desired materials ${ }^{[37]}$.

In a previous work ${ }^{[37]}$ we suggested the use of a C-ME tip in the tip-generation/substrate-collection for quantify the relative rates of parallel reactions. Here we show that the same method can be easily used in the determination of side reactions occurring in PEC-WS semiconductor materials and in their characterization under external potential and illumination.

Measurements with pulsed illumination will show the lightdriven $\mathrm{H}_{2}$ production on different photocathode materials and in particular the ratio $i_{\text {substrate }} / i_{\text {tip }}$ will provide the percentage of hydrogen production, thus the photofaradaic efficiency.

\section{Results and Discussion:}

The here described technique is a specific application of the so-called Tip Generation-Substrate Collection (TG-SC) mode. Briefly, the cavity (filled with the material of interest) produces the specie of interest (hydrogen or oxygen) and the Pt substrate works as an electrochemical probe for a quantitative determination of the material efficiency (see Figure 2).

Indeed, this method provides useful information to decouple concurrent reactions since:

$I_{\text {tip }}=I_{\mathrm{t}, \mathrm{c}}+l_{\mathrm{t}, 1}+l_{\mathrm{t}, 2}+l_{\mathrm{t}, 3}+\ldots$

where $I_{\text {tip }}$ is the tip overall current intensity, $I_{t, c}$ the tip capacitive current, $I_{f, 1,2,3}$ the faradaic currents of different electrochemical reactions:
1) $A+n e^{-} \rightarrow B$
e.g. $2 \mathrm{H}^{+}+2 \mathrm{e}^{-} \rightarrow \mathrm{H}_{2}$
2) $C+n ' e^{-} \rightarrow D$
e.g. $\mathrm{Cu}_{2} \mathrm{O}+2 \mathrm{e}^{-}+2 \mathrm{H}^{+} \rightarrow 2 \mathrm{Cu}+\mathrm{H}_{2} \mathrm{O}$
3) $\mathrm{E}+\mathrm{n}^{\prime \prime} \mathrm{e}^{-} \rightarrow \mathrm{F}$

and so on.

On the other side, the substrate current, $I_{\text {sub}}$, can be related to one or more of the products generated by reaction at the tip. For example,

$I_{\mathrm{sub}}=I_{\mathrm{s}, \mathrm{c}}+l_{\mathrm{f}, \mathrm{sub}}+l_{\mathrm{f},-1}$

where $I_{\mathrm{s}, \mathrm{c}}=$ substrate capacitive current, $I_{\mathrm{s}, \mathrm{sub}}=$ faradaic current relevant to any process occurring at the substrate independently on the presence of the tip, $l_{\mathrm{f},-1}=$ current due to the reaction-n consuming the product of reaction 1 ):
-1) $B \rightarrow X+n " ' e$
e.g. $\mathrm{H}_{2} \rightarrow 2 \mathrm{H}^{+}+2 \mathrm{e}$

It follows that, if both substrate and tip currents are corrected for background currents and if $n=n$ "' (that is, $\mathrm{X}=\mathrm{A})$ : 
$I_{\text {sub }} / l_{\text {tip }}=I_{\mathrm{f}, \mathrm{a}} /\left(l_{\mathrm{f}, \mathrm{a}}+l_{\mathrm{f}, \mathrm{b}}+l_{\mathrm{f}, \mathrm{c}}+\ldots\right)=\phi_{\mathrm{PEC}}$

that is the photo-faradaic efficiency as defined above by eq. 1 .

This method is here applied to the evaluation of $\phi_{\mathrm{PEC}}$ in the case of photo-anodes and photo-cathodes for water splitting, where the investigated reactions are the oxygen evolution reaction (OER):

$2 \mathrm{H}_{2} \mathrm{O} \rightarrow 4 \mathrm{e}^{-}+4 \mathrm{H}^{+}+\mathrm{O}_{2}$

and the hydrogen evolution reaction (HER):

$2 \mathrm{H}^{+}+2 \mathrm{e}^{-} \rightarrow \mathrm{H}_{2}$

Here both reported in acidic media.

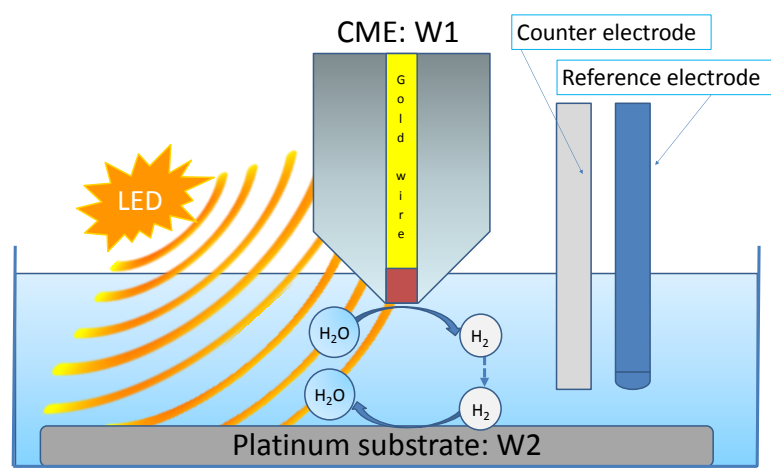

Figure 2: cell configuration for the evaluation of semiconductor activity and photodegradation process.

To confirm the effectiveness of the proposed method, we considered a model system composed by a conventional SECM platinum microdisk tip and platinum flat substrate $(A$ $=0.125 \mathrm{~cm}^{2}$ ) in aqueous $0.5 \mathrm{M} \mathrm{H}_{2} \mathrm{SO}_{4}$. The tip-to-substrate distance is set to $10 \mu \mathrm{m}$, as determined after the recording of negative feedback approach curves using the dissolved $\mathrm{O}_{2}$ as redox active species. The tip potential is varied from 0.9 to $-0.05 \mathrm{~V}$ vs the reversible hydrogen electrode (RHE). As shown in Figure 3 (black line), the current becomes increasingly negative below $0 \mathrm{~V}$ vs RHE. Under these conditions, we assume that only the reduction of $\mathrm{H}^{+}$to $\mathrm{H}_{2}$ occurs on a platinum electrode. The potential of the substrate electrode is kept constant at a potential that guarantees mass transport controlled $\mathrm{H}_{2}$ oxidation. The substrate and tip currents are simoultaneously recorded and are reported in Figure 3, from which it is evident that the ratio between the two currents at any potential is 1 . The whole amount of $\mathrm{H}_{2}$ produced at the tip is oxidized at the substrate, thus indicating that no side reactions are occurring.

The experiment was repeated at $\mathrm{pH} 7$ in $0.5 \mathrm{M}$ phosphate buffer solution, also adopted for the semiconductor measurements and the same results were obtained. In this condition, the HER becomes:

$2 \mathrm{H}_{2} \mathrm{O}+2 \mathrm{e}^{-} \rightarrow \mathrm{H}_{2}+2 \mathrm{OH}^{-}$

Eventually, using a cavity-microelectrode tip filled with $\mathrm{Pt} /$ Vulcan XC-72 $(28.6 \% \mathrm{w} / \mathrm{w})^{[38]}$, the results show that substrate and tip current intensities are equal. In this way, the absence of any influence from $\mathrm{pH}$ or the use of a cavity instead of a microdisk was confirmed as well as the capability of the system in recording $100 \%$ of the $\mathrm{H}_{2}$ produced at the cavity. It is important to state that the current reported here might be not sufficient to produce gas bubbles. Moreover, the enhanced mass transport, due to radial diffusion, leads to a rapid removal of $\mathrm{H}_{2}$ from the electrode surface. Indeed, previous work that adopted a similar approach ${ }^{[26]}$ for studying multireactional electrochemical interfaces clearly evidences the effect of bubbles.

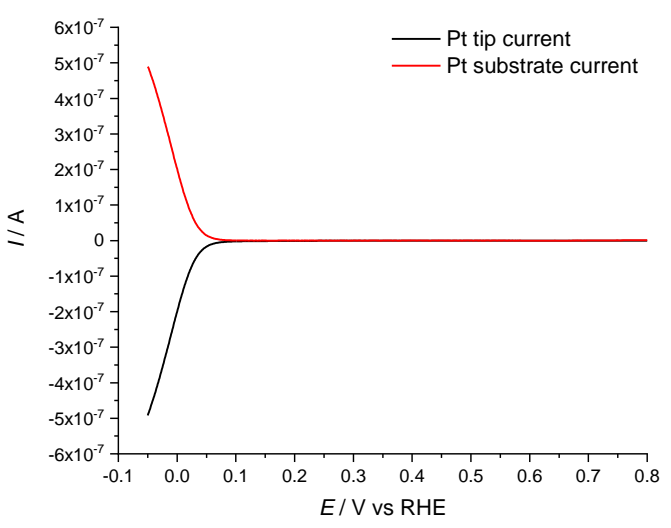

Figure 3: Control experiment adopting a Pt microdisk tip and a large $\mathrm{Pt}$ substrate in $\mathrm{H}_{2} \mathrm{SO}_{4}$ 0.5M. In black the Pt tip current of hydrogen evolution, in red the hydrogen oxidation performed by the Pt substrate. Counter electrode: Pt wire.

\section{$\mathrm{Cul} / \mathrm{CuO}$ photocathode}

The first studied semiconductor consists of a composite material recently introduced for its promising activity in both the HER and the OER ${ }^{[39]}$. It consists in a low-cost and high abundancy mixed copper oxide, which, however, may be still subdued to some modest photoreduction process 
when it initially enters in contact with light and potential bias. The material is here studied as a photocathode.

The C-ME tip is filled with the $\mathrm{Cul} / \mathrm{CuO}$ powder and used as working electrode 1 (WE1). WE1 potential is swept in the $0.45-0.7 \mathrm{~V}$ vs RHE potential range, thus avoiding the electrochemical HER while pulsing the LED light at about $0.2 \mathrm{~Hz}$ to highlight any difference between dark and light currents. On photocathodes, where the excited electrons are the minority charge carriers, the intense cathodic current (WE1) under light is due to hydrogen evolution and to unknown (or undetectable) side reactions. At the same time, the produced $\mathrm{H}_{2}$ is oxidized at the platinum substrate (WE2). In the dark there are no reactions at both electrodes, because the semiconductor is inactive and platinum has no hydrogen to consume. This electrodes configuration is schematically represented in Figure 2.

As working potential for the $\mathrm{H}_{2}$ oxidation the so-called "capacitive" region of $\mathrm{Pt}$ was chosen for the substrate electrode, to guarantee the absence of any parasite side reactions and have a clean platinum surface.

Figure 4 shows the results obtained with the $\mathrm{Cul} / \mathrm{CuO}$ powder inside the CME tip (WE1 = black line) and the relevant signal at the substrate electrode (WE2 = red line). Both tip and substrate current are background subtracted for the sake of clarity.

Figure 4 immediately unveils an interesting feature of this method, that is being time resolved. This is true even if one considers the time required for the tip products to reach the substrate, that is in the order of tens of ms. Indeed, we already demonstrated that $\mathrm{CuO}$ powders undergo an insitu partial reduction to $\mathrm{Cu}_{2} \mathrm{O}$, that is the active phase, then becoming stable ${ }^{[40]}$. In addition, electron-hole recombination can represent a significant contribution to the recorded photocurrent. In fact, by decreasing the potential (from $0.69 \mathrm{~V}$ to $0.46 \mathrm{~V}$ vs RHE), the tip photocurrent decreases, while $\mathrm{H}_{2}$ oxidation currents at the substrate remain constant. This is a clear evidence of the dynamics of the system. The tip current is higher than that of the substrate (photo-faradaic efficiency varies from 0.3 at $0.65 \mathrm{~V}$ to 0.9 at $0.50 \mathrm{~V}$ vs $\mathrm{RHE}$ ), meaning that the former testifies the reduction of the material within the cavity. In other words, a consistent part of the tip current is here related to undesired reactions and not solely to the HER.

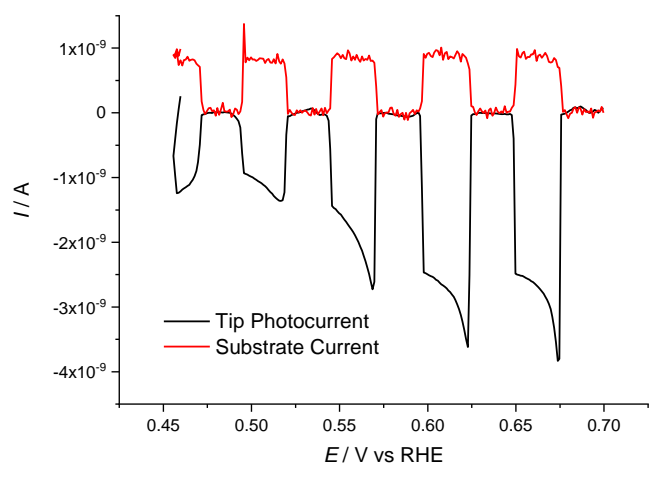

Figure 4. Linear sweep voltammetry at $10 \mathrm{mV} \cdot \mathrm{s}^{-1}$ in $\mathrm{pH} 7$ buffered solution under pulse illumination of a fresh powder. WE1: cavity filled with $\mathrm{Cul} / \mathrm{CuO}$. WE2: Pt substrate held at 0.79 V vs RHE. Counter: Pt wire. Reference: $\mathrm{Ag} / \mathrm{AgCl} 3 \mathrm{M}$. Lightsource: a $540 \mathrm{~nm}$ LED.

A closer look evidences that the tip-to-substrate current ratio is maximum after the cell is illuminated. Indeed, initial photo-current spikes (often observed in photoelectrochemistry just after every light/dark step) are well-known to be related to electron-hole recombination ${ }^{[41,42]}$. The absence of the same spike in the substrate current could be considered another proof of the robustness and of the effectiveness of the method.

Indeed, these observations are valid at the highest potentials, whereas at the lowest ones tip and substrate currents tend to converge to the same absolute value. Moreover, after the powder has been used for a sufficient time, it becomes stable, as evident from the data reported in Figure 5, that are recorded after keeping the tip at reducing potential for a few minutes, and in which tip and substrate current assume very similar values: the photofaradaic efficiency is 1 .

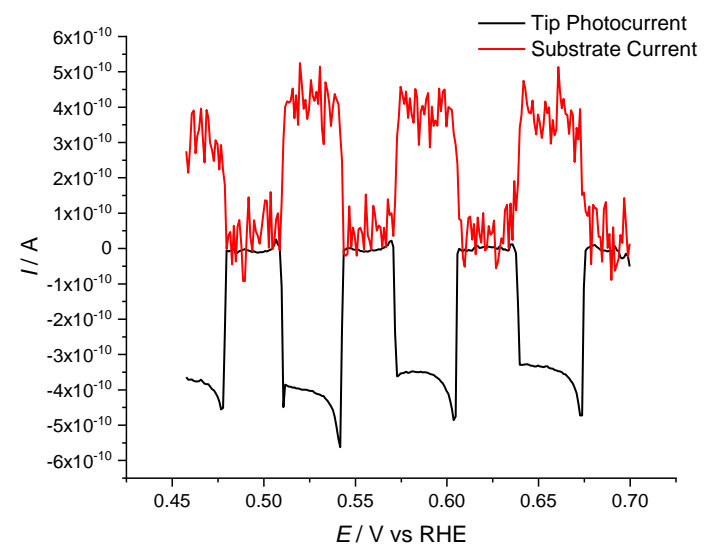


Figure 5. Linear sweep voltammetry at $10 \mathrm{mV} \cdot \mathrm{s}^{-1}$ in $\mathrm{pH} 7$ buffered solution under pulse illumination. WE1: cavity filled with $\mathrm{Cul} / \mathrm{CUO}$. WE2: Pt substrate held at $0.79 \mathrm{~V}$ vs RHE. Counter: Pt wire. Reference: $\mathrm{Ag} / \mathrm{AgCl} 3 \mathrm{M}$. Lightsource: a $540 \mathrm{~nm}$ LED

In order to confirm this conclusion, we extended the investigated potential window to further reduce the semiconductor. In Figure 6, the electrochemical reduction of the material onset is evident at about $0.2 \mathrm{~V}$ vs RHE (black line). While the tip current undergoes a dramatic increase, the substrate current exhibits the same alternate pattern. Hence, the tip signal is not related to $\mathrm{H}_{2}$ formation but only to reduction of the material in the tip.

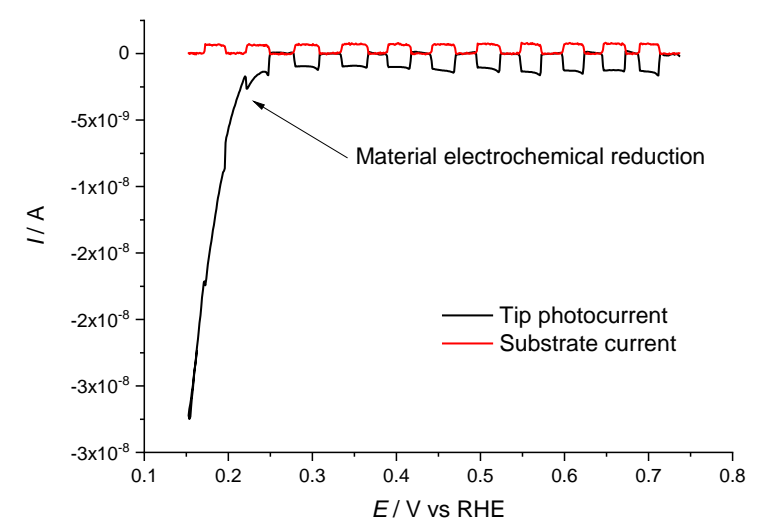

Figure 6. Linear sweep voltammetry at $10 \mathrm{mV} \cdot \mathrm{s}^{-1}$ in $\mathrm{pH} 7$ buffered solution under pulse illumination. WE1: cavity filled with Cul/CuO. WE2: Pt substrate held at $0.79 \mathrm{~V}$ vs RHE. Counter: Pt wire. Reference: $\mathrm{Ag} / \mathrm{AgCl} 3 \mathrm{M}$. Lightsource: a $540 \mathrm{~nm}$ LED

A further extension of the substrate potential window, as shown in Figure 7, leads to the electrochemical water reduction.

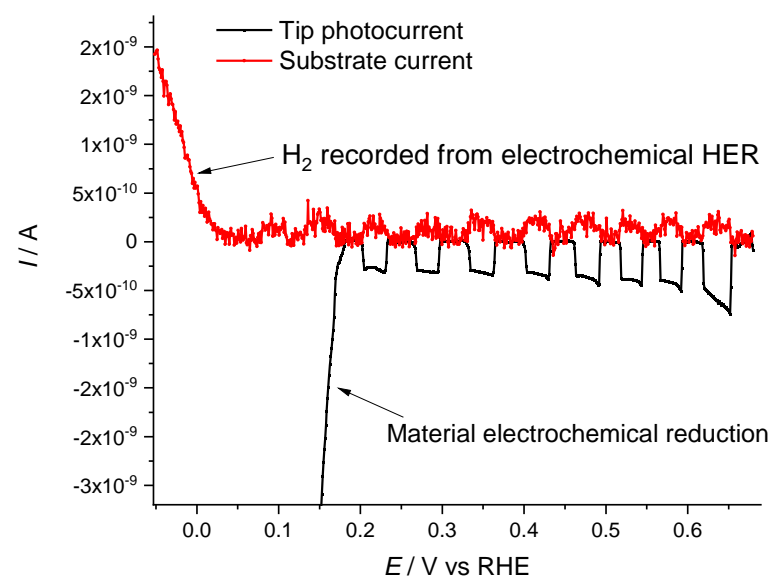

Figure 7. Linear sweep voltammetry at $10 \mathrm{mV} \cdot \mathrm{s}^{-1}$ in $\mathrm{pH} 7$ buffered solution under pulse illumination. WE1: cavity filled with Cul/CuO. WE2: Pt substrate. Counter: Pt wire held at $0.79 \mathrm{~V}$ vs RHE. Reference: $\mathrm{Ag} / \mathrm{AgCl} 3 \mathrm{M}$.
Data shown in Figures 6 and 7 demonstrate that the substrate (red line) is able to detect only the hydrogen fluxing from the tip. Indeed, the strong reduction recorded at the tip starting from $0.2 \mathrm{~V}$ have no influence on the substrate current in the dark. This means that in the potential region from $0.2 \mathrm{~V}$ to $0.1 \mathrm{~V}$ vs $\mathrm{RHE}$ the material degradation (electrochemical $\mathrm{CuO}$ and $\mathrm{Cu}_{2} \mathrm{O}$ reduction to $\mathrm{Cu}$ ) is the prevailing phenomenon. When the potential is negative enough to drive electrochemical $\mathrm{HER}$ on $\mathrm{Cu}_{2} \mathrm{O}$ and on $\mathrm{Cu}$ (Figure 7 below $0 \mathrm{~V}$ vs $\mathrm{RHE}$ ), also the substrate current witnesses it (Figure 7, red curve).

In order to conclude this section, it is worth commenting briefly on the absolute photocurrent values, we compared the geometric photocurrent density reported for the case of $200 \mu \mathrm{g}$ of $\mathrm{Cul} / \mathrm{CuO}$ deposited onto a conventional $1 \times 1 \mathrm{~cm}^{2}$ electrode lead a photocurrent of $0.1 \mathrm{~mA} \mathrm{~cm}^{2}$. ${ }^{[39]}$ Considering the current-to-mass resulting ratio and the estimated load of powder in the C-ME, we should expect a photocurrent that is about two orders of magnitudes higher that the ones observed in the present work. However, one has to consider that in the present case the monochromatic source illumination is not optimized and is highly diffused by the electrolyte and by the tip glass walls.

\section{Cul photocathode}

The second material tested is Cul, the precursor material for $\mathrm{Cul} / \mathrm{CuO}$, a semiconductor with $\mathrm{p}$-type character.

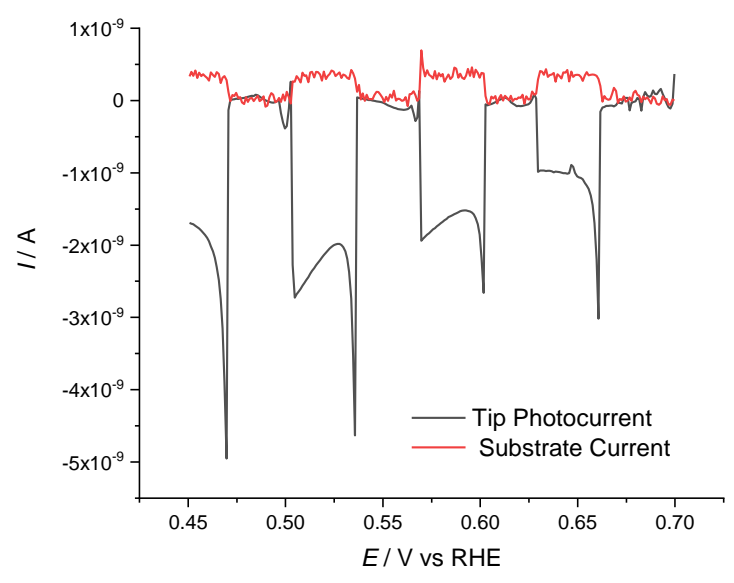

Figure 8. Two different experiments of $\mathrm{Cul}$ in buffer electrolyte $\mathrm{pH} 7$. Tip potential is scanned between 0.7 and $0.45 \mathrm{~V}$ vs RHE while substrate potential is held at $0.79 \mathrm{~V}$ vs RHE to oxidize the produced hydrogen during light pulsation at the frequency of $0.2 \mathrm{~Hz}$.

Figure 8 shows that the photodegradation contribution is higher than in the case of $\mathrm{Cul} / \mathrm{CuO}$ and that the hydrogen 
generation rate is quite low. This experiment clearly demonstrates that it is not possible to consider photocurrent as a direct measure of the material's performance, since only a portion of it is due to the HER. In this case $\phi_{P E C}$ is less than $10 \%$.

\section{Self-doped $\mathrm{TiO}_{2}$ photoanode}

$\mathrm{TiO}_{2}$ is a photoanode well-know for its long-term stability, thus allowing to further highlight the advantages of the here introduces method. $\mathrm{TiO}_{2}$ shows near $100 \%$ efficiency in oxygen production. This means that there are no side reactions occurring under oxygen evolution reaction conditions at the cavity-microelectrode tip (while oxygen reduction occurs on the platinum substrate), as shown in Figure 9, where $\phi_{\mathrm{PEC}}$ is almost $100 \%$. This can be considered as a further proof for the robustness of this method.

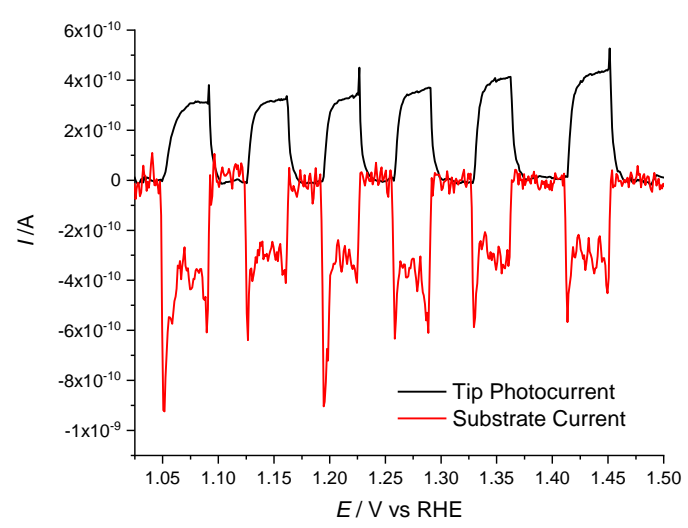

Figure 9. Exeriment with doped $\mathrm{TiO}_{2}$ inside the tip/cavity in $\mathrm{NaOH} 0.1$ $\mathrm{M}$ in the potential range between 1 and $1.5 \mathrm{~V}$ vs RHE. Substrate is canstantly polarized at $0.55 \mathrm{~V}$ vs RHE. Tip current of oxygen production (blue line) and substrate current of oxygen reduction (orange line).

\section{The role of the cavity's depth}

During this work, cavities of different depths were prepared to investigate on the role of the cavity's geometry on the final tip behavior. While the tip radius is almost constant (variation depends on the sealing of the glass tube around the gold wire), the cavity depth was varied from 24 to 46 $\mu \mathrm{m}$. The tips were then filled with a stabilized $\mathrm{Cul} / \mathrm{CuO}$ as test material in an aqueous phosphate buffer solution, $\mathrm{pH}$ 7.
Fig. 10a shows tip and substrate currents for three different tips and immediately suggests linear a trend as a function of the cavity depth.

Averaged current values sampled at the end of each light step are reported in Fig 10b versus the experimental values of the cavities' depth. The plot clearly demonstrates the existence of a linear relation between the cavity depth and the tip (and substrate) current. This confirms that all the material inside the cavity is active, being reached by both the light and the electrolyte. Considering the error bars, the photoefficiency, is $100 \%$ in all cases meaning that the depth of the cavity does not play a role in the measured photo-faradaic efficiency, at least up to $46 \mu \mathrm{m}$.

Figure 10 serves also to set important guidelines. Indeed, either too small or too deep cavities should be avoided, the former may lead to loss of material and to low current values, the latter may be too deep to be fully reached by the light.

a)

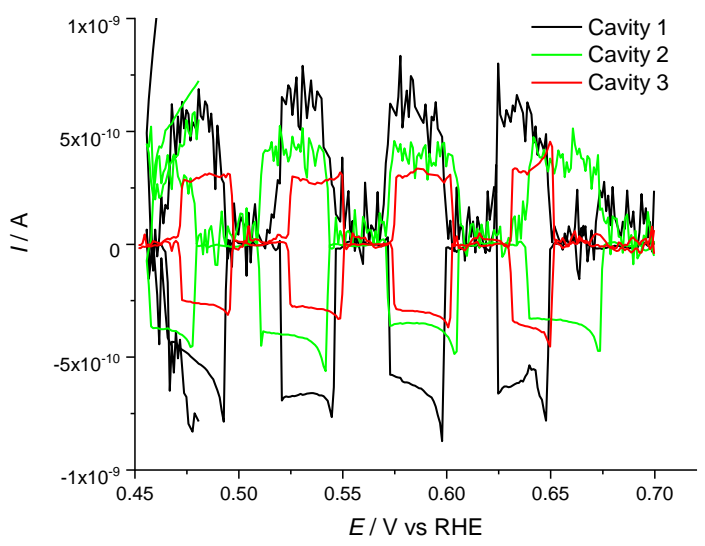

b)

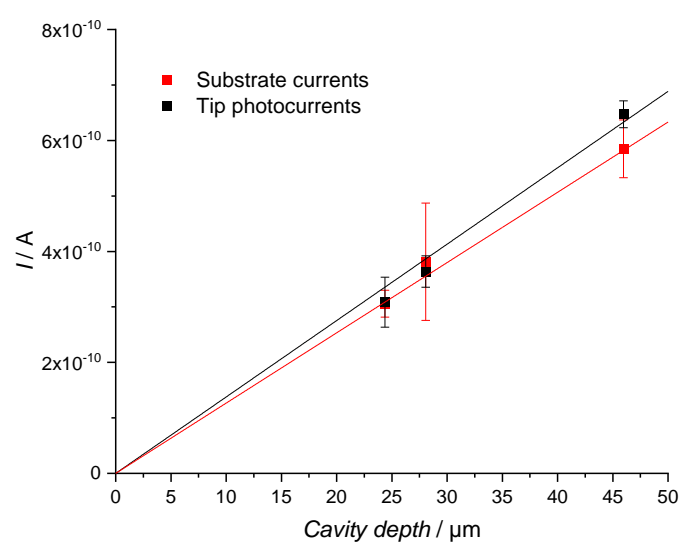

Figure 10. a) Different experiments with 3 different cavities containing $\mathrm{Cul} / \mathrm{CuO}$ in buffer electrolyte. Tip potential is usually scanned between 
0.7 and $0.4 \mathrm{~V}$ vs RHE while substrate potential is held at $0.79 \mathrm{~V}$ vs RHE to oxidize the produced hydrogen during light pulsation; b) Relationship between tip and substrate currents with cavity depths and their linear fitting. Tip has been filled with $\mathrm{Cul} / \mathrm{CuO}$ powder and immersed in buffered electrolyte at $\mathrm{pH}$ 7. Tip and substrate current are an average over at least 6 points from part a).

This results demonstrated that adopting deeper cavities leads to higher tip currents, thus allowing for a higher signal/noise ratio.

\section{Conclusion:}

In this paper we introduced a new method for decoupling the rate of desired $v s$ undesired photoelectrochemical reactions (photo-faradaic efficiency) as a function of the applied bias.

The use of the C-ME ensures a quickly and quantitative photoelectrochemical characterization of the material avoiding the influence of the conductive support or of any gluing agent (ionomers, etc.). Even considering the time require to the tip's reaction product to reach the substrate, that is in the order of tens of ms, the method is time resolved, since degradation phenomena occur in the order of $s$ or tens of $s$. Moreover, the method does not need any accumulation of the reaction product before the analysis and gives an immediate response to any perturbation of light and potential, as demonstrated, for example, in the case of electron-hole recombination current peaks.

In conclusion, we suggest this method as a fast and reliable solution both for a preliminary evaluation and fast screening of both newly synthetized photoactive materials as well as for more detailed characterization on well-known materials.

\section{Experimental Section:}

In all the synthesis and characterizations, MilliQ® water was used. All the reagents, if not explicitly declared, were purchased from Sigma Aldrich and used without any further purification.
Tip preparation: C-ME tip were prepared starting from $25 \mu \mathrm{m}$ radius gold wire (Goodfellow, 99.99\% purity) and glass capillary (O.D/I.D 1.5/0.75mm \#27-37-1, Frederick Haer \& Co, Bowdoinham, ME) previously cleaned with acetone in ultrasound bath. Once dried the gold wire is embedded in the capillary glass tube using a hot coil under vacuum. The Au disk is first exposed and then polished by using emery papers (400-800-1000-2400-4000 mesh) and alumina powders suspension in water (mean particle size 0.3 and $0.05 \mu \mathrm{m}$ ). The surface then is sharped until the desired $R G$ (ratio between tip and gold radius) value is reached (here between 4 and 6). Silver epoxy glue was used to connect a copper wire to the gold one in order to have the electric contact.

Cavity preparation: The procedure has been previously described ${ }^{[24,31,37]}$. Starting from a conventional microdisk SECM tip, the procedure consists in four different step; I) a very slow $\left(2 \mathrm{mV} \cdot \mathrm{s}^{-1}\right)$ cyclic voltammetry of the tip is performed in a $1 \mathrm{mM} \mathrm{Ru}\left(\mathrm{NH}_{3}\right)_{6} \mathrm{Cl}_{2}, 0.1 \mathrm{M} \mathrm{KClO}_{4}$ bath from 0 to $-0.4 \mathrm{~V}$ vs SCE. From the steady state current of the sigmoidal shape of the $\mathrm{CV}$, using the equation ${ }^{[24,31]}$,

$I_{\mathrm{ss}}=4 n F C_{\mathrm{b}} D r$

it is possible to define the real tip radius. II) The tip recess (cavity) is then generated in a $\mathrm{Cl}^{-}\left(0.5 \mathrm{M} \mathrm{HCl}, 0.5 \mathrm{M} \mathrm{H}_{2} \mathrm{SO}_{4}\right)$ bath using square wave voltammetry with pulsed currents density of 1.5 (for $100 \mathrm{~s}$ ) and $-7.5 \mathrm{~mA} \cdot \mathrm{cm}^{-2}$ (for $1000 \mathrm{~s}$ ). The cathodic polarization is used to expel the gold ions from the cavity thanks to the formation of $\mathrm{H}_{2}$ microbubbles. The number of total steps is calculated according to the desired cavity depth. III) Fast $\left(1 \mathrm{~V} \cdot \mathrm{s}^{-1}\right)$ cyclic voltammetry (500 cycles) in the same $\mathrm{Cl}^{-}$bath, between gold reduction and oxidation peaks, leads to a more homogeneous surface. IV) Finally, a slow cyclic voltammetry at $2 \mathrm{mV} \cdot \mathrm{s}^{-1}$ in the $\mathrm{Ru}\left(\mathrm{NH}_{3}\right)_{6} \mathrm{Cl}_{2}$ bath is performed to define the cavity depth, using the following equation ${ }^{[43]}$ :

$I_{S S}=\frac{4 \pi n F D r^{2} C_{b}}{4 L+\pi r}$

where $L$ is the cavity depth. The cavity is then washed with MilliQ® grade water and acetone, dried at $80^{\circ} \mathrm{C}$ and, after cooling, filled with the material of interest simply using the C-ME as a pestle, by tapping it for at least 5 times onto a small amount of powder onto a flat, hard surface.

Note that SECM tips are typically defined, among other parameters, by their $R G$ value, where $R G$ is the ratio between the tip radius with respect to the metal wire 
radius. In the present case, tips with RG between 5 and 6 are considered. However, we don't expect the RG value to significantly affect the effectiveness of the method except for the typical effects that it has in SECM: the lower the $R G$, the better (closer) is the approach of the tip to the substrate, because the overall diameter of the tip is small. Moreover, the lower the RG the more fragile the electrode is and the more time-consuming is its preparation. On the contrary, the higher the RG the longer the travel distance of the tip product to reach the solution bulk laterally and the higher the probability of the same substance of being consumed at the substrate. We believe that, in the present case, the selected value of $R G$ represents the better compromise.

SECM Setup: The setup is composed by 4 -electrodes in the configuration showed in Figure 2. There are two working electrodes, the tip and the substrate. The first one (WE1) is the cavity microelectrode filled with the material of interest. The second one (WE2) is large platinum $\left(A=0.125 \mathrm{~cm}^{2}\right)$ substrate. The larger area of WE2 compared to WE1 is used to ensure $100 \%$ collection efficiency of species produced at the tip. The setup is completed with a Pt counter electrode and a $\mathrm{Ag} / \mathrm{AgCl} 3 \mathrm{M}$ reference electrode in a double bridge (agar-agar plus the cell electrolyte).

The position in the 3-dimensions of the tip, in respect to the substrate, can be precisely controlled by using a stepped motor, thus setting the distance between WE1 and WE2 in the order of less than one tip radius. All TG/SC SECM measurements were performed at $10 \mu \mathrm{m}$ distance between substrate and cavity. The distance was evaluated measuring the current related to reduction of the couple $\mathrm{O}_{2} / \mathrm{H}_{2} \mathrm{O}$ presents in the electrolyte solution before bubbling with nitrogen, obtaining a negative feedback.

The oxygen reduction reaction likely occurs at the cavity bottom, i.e. at the metallic disk. The particular geometry of the microelectrode does not guarantee to record conventional negative feedback approach curves, still allow to detect the contact between the tip and the substrate at the end of the approach. This is testified by a rapid and discontinuous change of slope of the tip current. The tip is then retracted for the desired distance.

Once tip and substrate are correctly positioned, the solution was bubbled with $\mathrm{N}_{2}$ for at least 30 minutes, to exclude ORR during the measurements, and the cell was covered with Parafilm ${ }^{\circledR}$ to maintain the inert atmosphere above the electrolytic solution.

The tip and substrate potential are independently controlled using a bipotenziostat $\mathrm{CH}$ instruments bipotentiostat (model CH920). The potential of the $\mathrm{Pt}$ substrate was held constant at the value for hydrogen oxidation in the case of photocathodic material or at the value for oxygen reduction if a photoanodic powder is under study. The tip potential instead changes with time following a linear sweep voltammetry at $10 \mathrm{mV} \cdot \mathrm{s}^{-1}$ in a potential window specific for the material under pulsed illumination. The source of the light is a $3.5 \mathrm{~W}$ reflected light of high intensity green LED ( $\lambda=540 \mathrm{~nm}$, LED ENGIN).

The powder within the C-ME is illuminated by the LED, that is positioned on top of the cell, as close as possible to the electrolyte. Light diffuses in the entire setup and reaches the powder likely by both reflections on the Pt substrate surface and by transmission through the glass tip walls.

Each semiconductor studied required a proper electrolyte as described in the material section.

\section{Preparation of photomaterials}

Three different material are used here to prove the effectiveness of the proposed method:

Cul/CuO: a copper oxide material prepared by calcination of copper iodide in air atmosphere in the temperature 400 ${ }^{\circ} \mathrm{C}$ for 1 hour ${ }^{[39]}$. The obtained black powder was grinded in a mortar prior insertion in the cavity. The choice goes to this material because as any copper (I) based material it undergoes to photodegradation to metallic copper during the light driven water splitting, even if this material is quite stable. This material was studied in $0.5 \mathrm{M}$ disodium hydrogen phosphate +0.5 potassium dihydrogen phosphate buffer electrolyte $(\mathrm{pH} 6.86)$ in potential windows that starts from $0.7 \mathrm{~V}$ vs RHE reaching different potentials according to the desired experiment.

Cul: Copper iodide was synthesized according to the following procedure: $100 \mathrm{ml}$ of aqueous $0.01 \mathrm{M} \mathrm{Na}_{2} \mathrm{SO}_{3}$ were added to $60 \mathrm{ml}$ of $0.05 \mathrm{M} \mathrm{CuSO}_{4}$ while stirring. $300 \mathrm{ml}$ of aqueous $0.01 \mathrm{M} \mathrm{KI}$ were then added dropwise to the resultant green suspension. The obtained white precipitate was separated by centrifugation, washed 3 times with water and ethanol, and dried in air at about $80{ }^{\circ} \mathrm{C}$ for 8 
hours. This material was studied in the same electrolyte and potential window of $\mathrm{Cul} / \mathrm{CuO}$.

Self-doped $\mathrm{TiO}_{2}$ : hydrogenated self-doped titanium dioxide is obtained by commercial $\mathrm{TiO}_{2}$ anatase $(99.7 \%$ purity, $15 \mathrm{~nm}$ average particle size, Alfa Aesar) reduced at $500{ }^{\circ} \mathrm{C}$ for $1 \mathrm{~h}$ in flowing hydrogen $\left(50 \mathrm{~cm}^{3} \cdot \mathrm{min}^{-1}\right)$ inside a $\mathrm{U}$-shape Pyrex reactor. The result was a blue-grey powder (14 $\mathrm{nm}$ average particle size) that is stable for several weeks. This material was studied in $0.1 \mathrm{M} \mathrm{NaOH}$ between 1 and $1.5 \mathrm{~V}$ vs RHE. The synthesis was also described elsewhere $^{[2]}$.

\section{Conflict of interest:}

The authors declare no competing financial interest or other conflict of interest.

\section{Acknowledgments:}

The Authors thankfully acknowledge Università degli Studi di Milano (Piano di sostegno alla ricerca) for funding. A.V. is grateful to Universitá degli studi di Milano for founding his Ph.D fellowship. We are grateful to Dr. Alessandro Gallo (Stanford University) for having provided a sample of self-doped $\mathrm{TiO}_{2}$. We are thankful to Dr. Sara Morandi for the fruitful discussion.

\section{Keywords:}

Hydrogen evolution reaction (HER), Oxygen evolution reaction (OER), Photoelectrochemistry, Photocorrosion, Photoefficiency.
[13] A. Visibile, M. Fracchia, T. Baran, A. Vertova, P. Ghigna, E. Ahlberg, S. Rondinini, A. Minguzzi, J. Solid State Electrochem. 2019, DOI 10.1007/s10008019-04441-z.

[14] K. Katayama, S. Kuwahara, in (Eds.: A.A. Bakulin, R. Lovrincic, N. Banerji), 2016, p. 99230J.

[15] H. Kisch, Angew. Chemie - Int. Ed. 2013, 52, 812847.

[16] S. Wojtyła, T. Baran, Int. J. Hydrogen Energy 2019, 44, 14624-14634.

[17] H. Sudrajat, Mater. Res. Express 2018, 5, 065519.

[18] G. Liu, B. Sun, H. Li, Y. Wang, H. Zhao, J. Mater. Chem. A 2019, 7, 18529-18537.

[19] P. Rodenas, T. Song, P. Sudhagar, G. Marzari, H. Han, L. Badia-Bou, S. Gimenez, F. FabregatSantiago, I. Mora-Sero, J. Bisquert, et al., $A d v$. Energy Mater. 2013, 3, 176-182.

[20] Y. Bu, Z. Chen, W. Li, J. Yu, ACS Appl. Mater. Interfaces 2013, 5, 5097-5104. 
[21] Y. Tang, X. Hu, C. Liu, Phys. Chem. Chem. Phys. 2014, 16, 25321-25329.

[22] Z. Hens, W. P. Gomes, J. Phys. Chem. B 2000, 104, 7725-7734.

[23] H. Gerischer, J. Electroanal. Chem. Interfacial Electrochem. 1977, 82, 133-143.

[24] A. Minguzzi, C. Locatelli, O. Lugaresi, A. Vertova, S. Rondinini, Electrochim. Acta 2013, 114, 637-642.

[25] C. Locatelli, A. Minguzzi, A. Vertova, P. Cava, S. Rondinini, Anal. Chem. 2011, 83, 2819-2823.

[26] K. C. Leonard, A. J. Bard, J. Am. Chem. Soc. 2013, 135, 15890-15896.

[27] G. Castello, E. Biagini, S. Munari, J. Chromatogr. A 1965, 20, 447-451.

[28] Y. H. Su, S. H. Huang, P. Y. Kung, T. W. Shen, W. L. Wang, ACS Sustain. Chem. Eng. 2015, 3, 19651973.

[29] S. Uchiyama, H. Matsuura, Y. Yamawaki, Electrochim. Acta 2013, 88, 251-255.

[30] A. A. Dubale, A. G. Tamirat, H.-M. Chen, T. A. Berhe, C.-J. Pan, W.-N. Su, B.-J. Hwang, J. Mater. Chem. A 2016, 4, 2205-2216.

[31] A. Minguzzi, C. Locatelli, G. Cappelletti, C. L. Bianchi, A. Vertova, S. Ardizzone, S. Rondinini, J. Mater. Chem. 2012, 22, 8896.

[32] E. Guilminot, A. Corcella, M. Chatenet, F. Maillard, J. Electroanal. Chem. 2007, 599, 111-120.

[33] C. Cachet-Vivier, M. Keddam, V. Vivier, L. T. Yu, J. Electroanal. Chem. 2013, 688, 12-19.

[34] M. L. Tremblay, M. H. Martin, C. Lebouin, A. Lasia, D. Guay, Electrochim. Acta 2010, 55, 6283-6291.

[35] R. A. Rincón, A. Battistel, E. Ventosa, X. Chen, M. Nebel, W. Schuhmann, ChemSusChem 2015, 8, 560-566.

[36] J. Behnken, M. Yu, X. Deng, H. Tüysüz, C. Harms, A. Dyck, G. Wittstock, ChemElectroChem 2019, 6, 3460-3467.

[37] S. Morandi, A. Minguzzi, Electrochem. commun. 2015, 59, 100-103.
[38] A. Minguzzi, L. Montagna, A. Falqui, A. Vertova, S. Rondinini, P. Ghigna, Electrochim. Acta 2018, 270, 378-386.

[39] T. Baran, A. Visibile, S. Wojtyła, M. Marelli, S. Checchia, M. Scavini, F. Malara, A. Naldoni, A. Vertova, S. Rondinini, et al., Electrochim. Acta 2018, 266, DOI 10.1016/j.electacta.2018.02.023.

[40] T. Baran, S. Wojtyła, C. Lenardi, A. Vertova, P. Ghigna, E. Achilli, M. Fracchia, S. Rondinini, A. Minguzzi, S. Wojtyla, et al., ACS Appl. Mater. Interfaces 2016, 8, 21250-21260.

[41] M. Mokhtarimehr, S. A. Tatarkova, J. Opt. Soc. Am. B 2017, 34, 1705

[42] X. Qiao, C. Zhao, B. Chen, L. Luan, B. Hu, Org. Electron. 2014, 15, 1624-1630.

[43] A. M. Bond, D. Luscombe, K. B. Oldham, C. G. Zoski, J. Electroanal. Chem. Interfacial Electrochem. 1988, 249, 1-14.

\section{Table of content:}

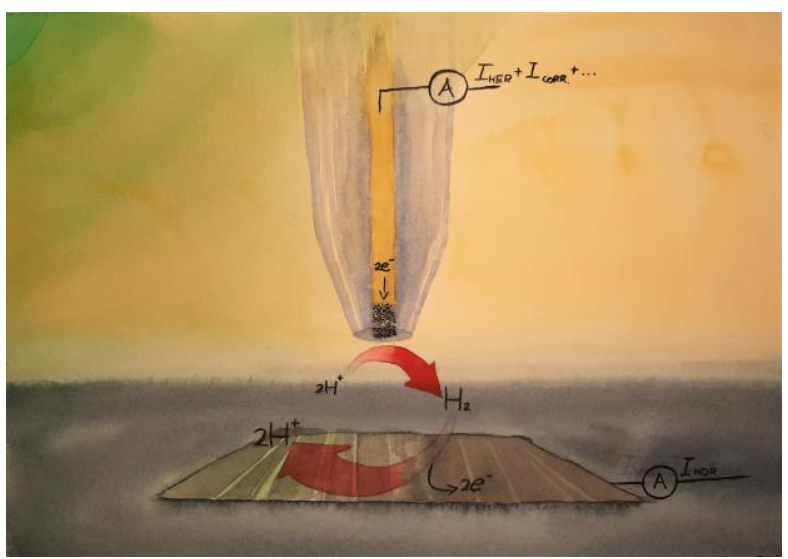

The article proposes a novel method to evaluate the photoefficiency of hydrogen (or oxygen) production on powder materials for photoelectrochemical water splitting. Scanning electrochemical microscopy used in Tip-generation /substrate-collection mode allows to discriminate between the desired electron transfer and recombination and/or undesired side reaction that may lead to material photodegradation. 